\title{
M otivações e fatores críticos de sucesso para o planejamento de sistemas interorganizacionais na sociedade da informação
}

\author{
H enrique Flávio Rodrigues da Silveira \\ D outorando em ciência da informação (UnB). M estre em ciência \\ da informação (UnB, 2001). Analista do Banco Central do Brasil \\ com atuação na área de planejamento e orçamento. \\ E-mail: henrique.silveira@ bcb.gov.br
}

\begin{abstract}
Resumo
O trabalho identifica, classifica e analisa as motivações e os fatores críticos de sucesso a ser considerados no processo de planejamento de sistemas interorganizacionais no setor público utilizando o ambiente Internet. A conclusão registra que a decisão de implementar sistemas interorganizacionais significa uma quebra de paradigma, por afastar-se da forma tradicional de gerenciamento de sistemas e passar a conviver com os benefícios (e riscos) do modelo colaborativo, com outros limites e novas regras.
\end{abstract}

Palavras-chave

Governo; Cidadania; Informação; Sistemas interorganizacionais; Fatores críticos de sucesso.

\section{Motivations and critical success factors for planning interorganizational systems in information society}

\begin{abstract}
This work identifies, classifies and analyzes the motivations and the critical success factors that should be considered in the planning process of interorganizational systems directed to the public sector using the Internet. The conclusion registers that the decision of implementing interorganizational systems means a paradigm break, since it introduces new limits and rules, and allows the breaking of the traditional way of systems' administration allowing the share of benefits (and risks) of the collaborative model.
\end{abstract}

\section{Keywords}

Government; Citizenship; Information; Interorganizational systems; Critical success factors.

\author{
"A I nternet ignora os três fundamentos do U niverso: \\ o do tempo, o do espaço e o da massa" \\ (Arno Penzias, Prêmio N obel deFísica)
}

\section{INTRO D U ÇÃO}

N este trabalho, foram identificados e classificados os fatores críticos de sucesso para o planejamento de sistemas de informação envolvendo várias organizações, com destaque para a oferta de informações e serviços públicos via Internet. Também foram identificadas e classificadas as razões que levam (ou podem vir a fazê-lo) as organizações à opção por sistemas interorganizacionais.

Para estudar a questão da motivação e dos fatores que podem determinar o sucesso na construção de sistemas interorganizacionais no setor público, inicialmente foi realizada uma revisão de literatura sobre sistemas interorganizacionais e suas aplicações na I nternet. A seguir, foi estudada a presença governamental na Internet e foram selecionados órgãos públicos que eventualmente poderiam participar de projetos envolvendo sistemas interorganizacionais no setor público no ambiente Internet.

Com o apoio do referencial teórico foram identificados os fatores críticos de sucesso e as motivações para a participação em sistemas interorganizacionais para o ambiente Internet. A partir de entrevistas dirigidas com os responsáveis pela área de planejamento de sistemas de informação dos órgãos selecionados, houve a classificação e a análise dos dados coletados.

Percebe-se que as organizações públicas utilizam cada vez mais a Internet para oferecer serviços e prestar informações à sociedade. A motivação para oferta de serviços einformações públicos no ciberespaço, por meio de sítios ( sites), faz parte do processo de reforma do Estado, que, entre outros pressupostos, prevêa existência demecanismos de controle dos organismos estatais por parte da sociedade. ConformePereira (1997), o princípio geral éque será preferível o mecanismo de controleque for mais geral, mais difuso, mais automático, o queé perfeitamente compatível com os fundamentos da Internet. 


\section{H enrique Flávio R odrigues da Silveira}

O Programa Sociedade da I nformação, lançado em 1999, apresenta, como um dos objetivos setoriais, 0 desenvolvimento de sistemas para integrar e ampliar ações de governo em benefício da cidadania, no novo "paradigma da sociedade da informação".

Segundo o documento de apresentação do programa, existem três tipos de organizações públicas na Internet:

a) oferecimento apenas de informações institucionais;

b) prestação de serviços relevantes dedownload (cópia a partir de um computador remoto) de informações para o usuário;

c) prestação de serviços públicos em tempo real e de forma interativa com o cidadão.

Assim, as organizações públicas utilizam a Internet como instrumento de aproximação com a sociedade, por meio da prestação de serviços e oferta de informações, ao mesmo tempo em que ampliam sua capacidade operacional e sua área de cobertura. 0 planejamento de sítios na I nternet, por parte das organizações públicas, deve incorporar a busca pelo interesse e necessidades dos cidadãos, e não apenas a redução de custo por transação. Jardim (1999)* ainda ressalva que as organizações públicas devem ir além da oferta de um simples folder eletrônico.

A realidade, entretanto, éque os sítios governamentais constituem um imenso arquipélago, formado por ilhas (sítios), que, ou não se comunicam entresi, ou o fazem por meio de pontes virtuais ( links) entre as ilhas, o que possibilita ao usuário navegar entre elas, mas, se houver a necessidade de organizar informações que estão distribuídas, caberá ao usuário a tarefa de reunir etentar padronizar o conjunto de informações coletadas. Assim, prevalecem sítios com administração individualizada, que no máximo oferecem a possibilidade de acesso a outros sítios, quando mais interessante seria uma visão de "continente", com sítios integrados de forma transparente para o usuário.

Para alcançar os propósitos de transparência e efetiva comunicação dos atos de governo esua repercussão, fazsenecessária maior integração das organizações públicas, no desenvolvimento de sítios que facilitem o exercício da cidadania. 0 usuário poderia acessar, a partir de um

\footnotetext{
* JARDIM, José M. Entre o vital e 0 virtual: 0 acesso aos arquivos
} públicos no Brasil, 1999. Não publicado. único sítio, um conjunto de informações e serviços de origem variada, destinados a um mesmo público, sem a utilização de ligações ( links) entre os sítios originais de várias organizações.

Contudo, o processo de desenvolvimento e gerência de sítios cooperativos traz implicações importantes para o processo de concepção, desenvolvimento e gerência de sistemas de informação. Questões como design, segurança, compatibilidade (hardware e software), diversidade de metodologia e de regras para publicação devem ser consideradas.

Para estudar o tema dos portais cooperativos no setor público - sítios com oferta de serviços e informações de origem variada, com o propósito de atender às necessidades dos usuários de forma integrada - , optouse pela delimitação da pesquisa, que enfoca o estudo das motivações e dos fatores críticos de sucesso para o desenvolvimento de sistemas interorganizacionais, que darão suporte informacional aos portais cooperativose permitirão que haja um novo tipo de presença de organizações públicas na Internet, a de portais interinstitucionais com informação integrada sobreáreas específicas de interesse do cidadão, das organizações e da sociedade.

\section{CONEXÃO COM A CIÊNCIA DA IN FO R M AÇÃO}

A ciência da informação, ciência de caráter interdisciplinar, tem por objeto o estudo das propriedades gerais da informação (natureza, gênese e efeitos). Assim, em pesquisas que abordam o tema da informação, a ciência da informação contribui com estudos das necessidades informacionais, processos de interação, sistemas de informação e estudo do uso da informação.

Também contribui para o planejamento de sistemas interorganizacionais, a partir de estudos e técnicas referentes ao ciclo informacional (figura 1, a seguir), a ser aplicado em um contexto no qual diferentes expectativas e culturas organizacionais irão interagir.

Sobre a importância da ciência da informação, Vickery \& Vickery (1989) afirmam que ela está identificada com o estudo da comunicação da informação na sociedade, o que pode significar efetiva contribuição para a facilitação dos processos de transferência de informações e para a construção da cidadania. A participação de profissionais da informação também

Ci. Inf., Brasília, v. 32, n. 2, p. 107-124, maio/ago. 2003 
é coerente com o princípio da multidisciplinaridade que deve reger a construção de sistemas de informação, especialmenteno caso de sistemas interorganizacionais.

0 estudo das motivações e dos fatores críticos de sucesso dos sistemas interorganizacionais no setor público é importante, pois a existência de tais sistemas facilitaria não somente 0 acesso dos cidadãos aos produtos e serviços governamentais, mas também 0 entendimento sistêmico da ação do governo, passando da visão de "arquipélago" (sítios como ilhas) para uma visão de "continente" (sítios integrados deforma transparente para o usuário).

Segundo M oresi (2000)*, as motivações para o planejamento de sistemas de informação podem ser classificadas de acordo com sua natureza:

a) conceitual - o planejamento de sistemas de informação decorre do exercício normal da atividade de gestão da organização;

b) prática - o planejamento de sistemas de informação decorre do reconhecimento etentativa de resolução pela organização de problemas de ordem prática no seu sistema de informação;

c) estratégica - o planejamento desistemas de informação decorre da procura de oportunidades pela utilização do sistema de informação e das tecnologias de informação associadas.

O s resultados esperados com o planejamento de sistemas de informação contemplam o alinhamento do sistema de informação com as necessidades da organização, a obtenção de vantagens competitivas pela utilização de tecnologia da informação, o comprometimento da alta direção da organização, a previsão das necessidades do sistema de informação e o estabelecimento de políticas e caminhos de desenvolvimento para a tecnologia da informação, em um contexto de permanente sintonia com 0 ambiente.

Em todo esse processo de monitoramento ambiental, as organizações selecionam, organizam, armazenam, recuperam e distribuem informação. Sobre as formas como as organizações utilizam a informação, Choo (1996) define três finalidades básicas:

\footnotetext{
* M ORESI, Eduardo. G estão da informação. Brasília : U niversidade de Brasília, 2000. Não publicado.

Ci. Inf., Brasília, v. 32, n. 2, p. 107-124, maio/ago. 2003
} a) para fundamentar o processo decisório - nesse caso,
a informação atua como um redutor da incerteza;

b) para interpretar o ambiente, reduzindo a equivocação;

c) para criar conhecimento novo.

Admite-seque fatores dos ambientes físico, econômico, social e institucional, assim como os recursos humanos e tecnológicos, determinam e influenciam os sistemas de informação. 0 processo demonitoramento ambiental permite a identificação, entre esses fatores, daqueles considerados críticos para o sucesso do planejamento, desenvolvimento e gerência de sistemas de informação. Essa identificação pode ser feita com o auxílio da técnica de fatores críticos de sucesso (Rockart, 1979).

Rockart (1979), em reconhecido estudo sobre a definição de necessidades informacionais para altos executivos, propôs uma nova metodologia, que contemplava a utilização da técnica defatores críticos de sucesso, como determinante para que os sistemas de informações gerenciais atendessem aos objetivos para os quais foram planejados.

O s fatores críticos de sucesso, segundo Rockart, podem ser estudados de acordo com o seu relacionamento com as áreas ou processos da organização. Alguns fatores críticos desucesso dizem respeito ao negócio da empresa, ao ramo de atividade em que ela atua. O utros podem ser atribuídos de forma geral aos vários componentes da organização, enquanto outros são específicos de determinadas unidades organizacionais.

A partir das considerações feitas sobre fatores críticos de sucesso, é reconhecida a necessidade de identificálos em número reduzido - alguns autores sugerem não 


\section{H enrique Flávio R odrigues da Silveira}

mais que uma dezena (Stollenwerk, 2000) ${ }^{*}$, a fim de evitar a dispersão que poderia ocorrer com o registro de um número elevado de fatores. Porém, uma vez identificado, um fator crítico de sucesso pode ser desdobrado de forma a contemplar os diferentes processos eáreas envolvidas, observadas as relações de pertinência. A essa propriedade de desdobrar os fatores críticos de sucesso, dá-se o nome de arborescência.

A dinamicidade admitida para o conjunto de fatores estratégicos identificados decorre dos impactos provocados pelas mudanças nos ambientes interno e externo da organização. Tais mudanças devem ser monitoradas e avaliadas e podem acarretar alterações no conjunto de fatores críticos de sucesso, que podem significar inclusão, exclusão ou alterações de importância.

Sobre o assunto objeto deste trabalho - motivações e fatores críticos de sucesso para o planejamento de sistemas interorganizacionais no setor público utilizando o ambiente Internet - , admite-se a existência deinúmeros aspectos que devem ser considerados para projetos e sistemas do tipo em estudo. Para isso, a utilização da técnica de fator crítico de sucesso permitirá maior atenção àqueles aspectos considerados mais relevantes e decisivos para o sucesso de iniciativas de sistemas interorganizacionais.

Este trabalho encontra apoio na ciência da informação e também contribui para essa área do conhecimento, por incorporar, apresentar e propor análises relativas à gestão da informação, que, conforme documentado, apóia-se cada vez mais em sistemas que envolvem mais de uma organização em sua concepção, planejamento, desenvolvimento, gerência e execução: os sistemas interorganizacionais.

D efine-se gestão da informação como sendo a aplicação de princípios administrativos à aquisição, organização, controle, disseminação e uso da informação para a operacionalização efetiva de organizações de todos os tipos (Wilson, 1997). 0 principal objetivo da gestão da informação é identificar e potencializar recursos informacionais de uma organização ou empresa, ensinando-a a aprender e adaptar-se às mudanças ambientais.

\footnotetext{
*ST O LLENWERK, M. F. O método dos fatores críticos de sucesso e sistemas de inteligência competitiva. In: CURSO DE INTELIGÊN CIA COM PETITIVA, 3., 2000, Brasília, DF. Não publicado.
}

\section{SISTEMAS, SISTEMAS DE INFORMAÇÃO E CIÊNCIA DA IN FORMAÇÃO}

Bertanlanffy (1977), em sua obra sobre a Teoria G eral dos Sistemas, define sistema como um complexo de elementos em interação, ea engenharia de sistemas como sendo o planejamento, traçado, evolução e construção de sistemas de modo científico. Admitindo a crescente mecanização, o autor ainda registra que, independentemente da opção por considerar positiva a expansão do conhecimento ou temerário o risco de controle social, a informatização dos sistemas é um fato e por isso precisa ser estudada.

Apesar de freqüentemente identificada com a teoria de sistemas, a cibernética éapenas uma parte da teoria geral, porque trata especificamente da teoria dos mecanismos de controle na tecnologia e na natureza, fundada nos conceitos de informação e retroação. Os sistemas cibernéticos são um caso especial, embora importante, dos sistemas queapresentam auto-regulação, em função de atuarem como sistemas abertos capazes de alcançar um estado de organização "superior", devido à informação introduzida no sistema.

Shimizu (1994), Bio (1996) e Cornachione (1998) apresentam conceitos de "sistema" que seguem a linha traçada por Bertanlanffy. Já o estudo de sistemas de informação revela uma profusão de terminologia e conceitos muitas vezes conflitantes entre si. M oresi (2000) apresenta as principais razões para essa situação:

a) o caráter marcadamente multidisciplinar dos sistemas de informação;

b) o pouco tempo de maturação dos sistemas de informação como ferramenta de gestão empresarial;

c) deformações e má aplicação intencional de terminologia econceitos, por razões comerciais ou de marketing;

d) elevado número de contribuições e comunicações esparsas, sem configurar escolas de pensamento.

Ein-D or \& Segev (1986), Saviani (1998), Bio (1998) e Albertin (1999), com pequenas variações, definem sistema de informação como um sistema para a coleta, armazenamento, recuperação e processamento de informações queéusado (ou poderia sêlo) para a tomada dedecisão. Registre-seo paralelo óbvio que se pode traçar com o ciclo informacional, objeto de atenção da ciência da informação. O s autores ainda destacam 
que um sistema de informação não é necessariamenteautomatizado, até porque sistemas de informações já existiam muito antes da invenção do computador.

Para Buckingham ( apud M oresi, 2000), um sistema de informação é um sistema que reúne, guarda, processa e faculta informação relevante para a organização, de modo quea informação esteja acessível e útil para aqueles que dela necessitam, incluindo gestores, funcionários, clientes. Assim, pode-se afirmar que é a utilidade da informação que indicará seu valor, que dependerá do contexto em queéutilizada. A utilidade pode ser percebida, entre outros fatores, pela melhoria da produtividade (eficiência), melhoria da qualidade na tomada de decisão, aumento no desempenho das tarefas (eficácia) eaumento na curva deaprendizagem organizacional.

Yourdon (1990) afirma que um sistema de informação pode existir independentemente da presença de processamento eletrônico de dados e que é preciso estudar aTeoria G eral dos Sistemas para que os sistemas de informação sejam construídos de forma a atender aos requisitos da estabilidade e confiabilidade. Buscar a essência dos sistemas é o desafio a ser vencido pelos profissionais envolvidos com o planejamento desistemas deinformação.

D essaforma, um sistema aberto podetender "ativamente" paraum estado deorganização superior, isto é, pode passar de um estado inferior de ordem a um estado superior de ordem, devido às condições do sistema eà internalização da informação. Bertanlanffy (1977) afirma queesse"estado deorganização superior" acontecedevido à aprendizagem, isto é, à informação introduzida no sistema.

Para Cruz (1998), tecnologia da informação é todo e qualquer dispositivo que tenha a capacidade de "tratar dadose/ ou informação", tanto deforma sistemática quanto esporádica, quer essa capacidadeesteja aplicada no produto ou no processo. Yourdon (1990) define tecnologia da informação como sendo o conjunto de equipamentos e suportes lógicos ( hardware esoftware), que permitem executar tarefas como aquisição, transmissão, armazenamento, recuperação eexposição dedados- nesta colocação há clara analogia com o ciclo informacional. Já para Albertin (1999), tecnologia de informação étudo aquilo com o que podemos obter, armazenar, tratar, comunicar etornar disponível a informação.

Ci. Inf., Brasília, v. 32, n. 2, p. 107-124, maio/ago. 2003
N ovamente temos uma analogia direta com o ciclo informacional. Pode-seafirmar queessa recorrência existe por causa da clara identificação do objeto de estudo da ciência da informação - a informação e o trabalho com a informação - com os processos de gestão em uma organização, que são apoiados por sistemas de informação. Assim, a gestão da informação (aquisição, armazenamento, análise e uso) provêa estrutura para o suporte ao crescimento e desenvolvimento de uma organização inteligente, voltada para o aprendizado, que seadapta, responde ese antecipa a exigências enovidades do ambiente.

Há, entretanto, cada vez mais sistemas de informação que ultrapassam as fronteiras de uma organização. São os sistemas interorganizacionais, conceito que estudaremosa seguir.

\section{SISTEM AS INTERORG AN IZACION AIS}

O s sistemas distribuídos, ou DDP (distributed data processing), que tinham seu processamento descentralizado dentro da organização, são os precursores dos sistemas interorganizacionais, que incorporaram parte dos requisitos tecnológicos desenvolvidos para os sistemas distribuídos (Claver; G arcia \& Gonzaléz, 2001), conforme mostra a figura 2.

Com relação a sistemas interorganizacionais, a maioria dos autores pesquisados utiliza o termo para definir relações entre duas ou mais organizações. Clarke(1992), porém, argumenta que sistemas que atendem a mais de duas organizações deveriam ser classificados como "multiorganizacionais".

Para efeito deste estudo, entretanto, será utilizado o termo consagrado na literatura, "sistemas interorganizacionais", para representar os sistemas deinformação que integram, 


\section{H enrique Flávio R odrigues da Silveira}

total ou parcialmente, os processos de negócio de duas ou mais organizações. Já "sistema de informação", ainda para o propósito deste trabalho, pode ser definido como sendo o conjunto de dados, procedimentos e canais de comunicação quepermitea estruturação da informação para 0 atendimento das necessidades dos processos decisórios em vários níveis.

Sistemas interorganizacionais ainda se referem à infraestrutura de computadores e telecomunicações desenvolvida, operada e/ ou utilizada por duas ou mais empresas com o propósito de trocar informações que apóiam processos de negócio ( Li \& Williams, 1999). As organizações podem ser fornecedores eclientes de uma mesma cadeia produtiva, parceiros estratégicos e até mesmo competidores.

Sistemas interorganizacionais podem ser classificados sob diversos aspectos. Claver, G arcia \& G onzaléz (2001) sugerem que os tipos de padrões utilizados e 0 relacionamento entreas organizações podem ser critérios válidos para classificação. Segundo o tipo de padrão utilizado, os sistemas interorganizacionais podem ser classificados em dois grupos:

a) padrões abertos - adotam padrões abertos em sua operação, seja em escala nacional ou internacional, como o padrão E difact para sistemas de intercâmbio eletrônico dedocumentos(EDI);

b) sistemas proprietários - adotam padrões particulares, desenvolvidos especificamente para determinado grupo de empresas ou softwares.

Em função da crescente necessidade de intercâmbio, há resistência cada vez maior entre as organizações quanto à adoção de sistemas proprietários etambém acentuada procura por padrões abertos, com aceitação internacional, em busca de soluções que facilitem a integração entre organizações esistemas.

Já a classificação com base no relacionamento entre as organizações apresenta quatro possibilidades:

a) sistemas interorganizacionais para mercados complementares - nessecaso, as organizações participantes não são competidoras entresi; oferecem serviços eprodutos complementares eutilizam a vantagem proporcionada pelos sistemas interorganizacionais para atuar no mercado. Como exemplo, pode-se citar a associação entrehotéis, locadoras ecartões decrédito, que compartilham informações sobre clientes comuns, com aumento nas possibilidades de negócio eredução de custos; b) sistemas interorganizacionais intra-setoriais - as organizações participantes são competidoras entresi, mas há vantagen n na associação em função da racionalização de custos a partir da utilização de uma infra-estrutura compartilhada de tecnologia da informação. Centrais de informações sobre créditos e os caixas automáticos utilizados por clientes de diversos bancos são exemplos nessa categoria;

c) sistemas interorganizacionais que conectam clientes e fornecedores - são os casos mais freqüentes; desenvolvidos entre organizações que integram uma mesma cadeia de valor em uma indústria ou ramo da indústria;

d) sistemas interorganizacionais direcionados pelos fornecedores de tecnologia da informação - uma empresa de tecnologia pode criar um novo produto e prover o arcabouço tecnológico para que seus clientes possam desenvolver seus negócios na nova plataforma.

Dependendo do tipo de informação compartilhada, Claver, Garcia \& Gonzaléz (2001) destacam que é possível classificar os sistemas interorganizacionais em primários e avançados. Primários seriam os sistemas menos sofisticados, que apenas habilitam troca de informações básicas entre os participantes.

Já em sistemas avançados, os participantes estão habilitados tanto a compartilhar informações, quanto a alterar dados de outras organizações. N esse tipo de sistema, há também a possibilidade de sugerirem alterações no processo produtivo de um parceiro, em função de necessidades operacionais ou de mudança de estratégia denegócios.

A questão sobre a compulsoriedade na integração de sistemas interorganizacionais pode levar a uma outra forma de classificação: sistemas interorganizacionais de adesão compulsória e sistemas interorganizacionais de adesão voluntária.

Claver, Garcia \& G onzaléz (2001) definem quesistemas interorganizacionais deadesão compulsória são aqueles em que a existência do sistema funciona como uma barreira à entrada de novos competidores, indicando que, para fazer parte daquelemercado, a adesão ao sistema interorganizacional existente não é uma opção para a organização. Em contrapartida, no caso dos sistemas interorganizacionais de adesão voluntária, não há restrição à participação no mercado pelo fato de não fazer parte do sistema existente.

Ci. Inf., Brasília, v. 32, n. 2, p. 107-124, maio/ago. 2003 


\section{M otivações e fatores críticos de sucesso para o planejamento de sistemas interorganizacionais na sociedade da informação}

A partir de estudo sobre as fontes de informação externa, Choo (1995) apresenta o conceito de "cadeia alimentar informacional", que se refere ao fato de que as várias fontes "alimentam-se" umas das outras, formando diversas cadeias alimentares inter-relacionadas, atéchegar ao consumidor final da informação.

D e forma geral, sistemas interorganizacionais requerem não apenas o desenvolvimento de infra-estrutura de tecnologia apropriada, mas também o reconhecimento do papel das empresas na cadeia produtiva ede aspectos como estrutura evalores.

Sistemas interorganizacionais são algumas vezes denominados redes entre empresas ( inter-firm networks) ou sistemas de informação entre empresas (inter-firm information systems). Segundo Li \& Williams (1999), os sistemas interorganizacionais podem ser divididos em três categorias:

a) utilização de tecnologia proprietária por grupos fechados;

b) utilização de tecnologia aberta por grupos fechados;

c) utilização de sistemas abertos baseados em mídias abertas como a I nternet.

Os sistemas interorganizacionais do primeiro grupo apresentam como característica a existência de uma interface proprietária para um grupo de empresas. Caso alguma outra organização venha a fazer parte do grupo, terá de investir na tecnologia (hardware e software) específica que está sendo utilizada pelo grupo. A utilização desistemas interorganizacionais dessa categoria podeestar vinculadaà proteção do mercado, à falta de padrões quando o sistemafoi desenvolvido, ou a necessidades de segurança não providas por sistemas abertos.

A segunda categoria é a mais difundida e contempla os sistemas desenvolvidos em padrões e protocolos abertos. Assim como os sistemas interorganizacionais da primeira categoria, nesse caso também os componentes do grupo são previamente conhecidos, configurando o que Li \& Williams (1999) chamam deelectronic alliance (aliança eletrônica).

A terceira categoria tornou-se possível com a Internet, devido à possibilidade de conexões entre um número não predeterminado de parceiros: é o electronic market (mercado eletrônico). N esse novo ambiente, há possibilidades reduzidas de bloquear mercados a novos competidores, eé praticamente impossível impedir um cliente de ter acesso a novos fornecedores.
ParaCrook \& Kumar (1998), sistemas interorganizacionais (IOS - interorganizational systems) são sistemas de informação desenvolvidos e utilizados por duas ou mais organizações que trabalham em rede. Esses sistemas de informação compartilhados, nos quais a informação é coletada, processada edistribuída, não apenas para uma, mas para várias organizações, são apoiados em intensa utilização de tecnologia da informação e devem assegurar eqüidade da cooperação e nos resultados, em um relacionamento do tipo winner-to- winner (algo como "ganha-ganha") (Claver, G arcia\& G onzaléz, 2001).

Ainda sobre a cooperação para o desenvolvimento e gerência de sistemas de informação, há um campo de pesquisa denominado CSCW (Computer Supported Cooperative W ork), ou "trabalho cooperativo apoiado por computador", também chamado groupware, que trata das necessidades tecnológicas para o desenvolvimento de trabalhos em grupo. As ferramentas degroupware devem permitir que pessoas distribuídas geograficamente possam se comunicar e compartilhar informações.

U m outro autor (Ciborra, 1993) considera queo conceito de groupware inclui dois elementos distintos: o group, de caráter socioorganizacional, que é uma forma de trabalho coletivo, envolvendo colaboração e compartilhamento, e o ware, representando 0 artefato ou ferramenta. 0 group ware conecta, então, dois mundos - um humano e um artificial -, e pode se constituir importante instrumento de aprendizado e transformação, muito mais que apenas uma eficiente ferramenta decoordenação.

N esse sentido, D rucker, já em 1971, afirmava que o teste de adequada utilização de tecnologia da informação virá do tempo de trabalho liberado para que os executivose demais profissionais possam fazer relações diretas e pessoais, libertando o ser humano da preocupação com a informação e lhe permitindo trabalhar com a comunicação. N esse novo ambiente, longe de serem afastadas as interações pessoais, estas são incrementadas pelo trabalho em grupo com auxílio da rede (internetworking).

Cresce, então, a necessidade de definição e implementação de sistemas que integrem processos e informações de várias organizações. Em especial no setor público, admite-se que as tecnologias hoje existentes podem facilitar a interação do governo com a sociedade. 


\section{H enrique Flávio R odrigues da Silveira}

\section{PRESEN ÇA G OVERNAMENTAL NA INTERNET}

No Brasil, o Programa Sociedade da Informação apresenta, como um dos objetivos setoriais, 0 desenvolvimento de sistemas para integrar e ampliar ações de governo em benefício da cidadania, no novo paradigma da sociedade da informação. O s conteúdos ofertados pelos governos (federal, estadual e municipal) devem, progressivamente, facilitar o exercício da cidadania. N esse sentido, Beniger (1986) afirma que o controle de qualquer sistema está relacionado ao processamento distribuído e ao acesso generalizado.

Além da redução de custos por transação, outros custos são alvo das atenções do governo. Segundo a Agência $\mathrm{N}$ acional de Telecomunicações (Anatel), a multiplicidade de redes de telecomunicações mantidas pelos diversos órgãos de governo, distribuídos nas diferentes esferas dos poderes Executivo, Legislativo e Judiciário, geram custos que poderiam ser reduzidos se houvesse maior integração entre as redes.

Para além dos custos financeiros, existe a questão da racionalização e integração das ações de governo, que gera custos e desperdícios difíceis de mensurar. Seria preciso utilizar de forma mais eficiente e de modo exemplar a infra-estrutura detelecomunicações, essencial para que o governo desenvolva suas atividades finalísticas.

0 desempenho da burocracia estatal deve ser melhorado pela otimização dos processos e diversificação dos meios para prestação de serviços. Essa melhoria não pode acontecer sem modernas tecnologias de criar, gravar, armazenar e processar informações (Beniger, 1986). O u seja, sem que o ciclo informacional esteja no centro das atenções em processos de desenvolvimento da oferta de serviços e informações à sociedade, praticando, de fato, o que foi definido como gestão da informação (Wilson, 1997).

Em 2000, Silveira desenvolveu um guia sobre a presença do governo brasileiro na I nternet, com concentração no Poder Executivo federal. A partir dos levantamentos feitos para a elaboração do guia e de contatos realizados com órgãos de coordenação superior, constatou-se aparente falta de controle sobre sítios governamentais e informações e serviços ofertados.

M esmo não sendo exaustivo, o guia buscou representar todas as áreas de atuação do Poder Executivo. Foi elaborada uma lista de grandes assuntos (áreas de atuação do governo - agricultura, saúde, ciência, tecnologia e outras) e realizada pesquisa para localizar sítios representativos daqueles segmentos de ação. 0 guia representa interessante documento sobre a presença do governo brasileiro na Internet e poderia ser útil como auxílio àqueles que precisam conhecer melhor o que neste trabalho foi denominado "arquipélago" formado pelas ilhas (sítios) de organizações públicas.

O próprio governo federal, reconhecendo a necessidade de maximizar sua presença na Internet, vem intensificando os esforços para organizar e disciplinar a questão. As possibilidades de utilização da I nternet para ampliar a interação do governo com a sociedadee para combater a exclusão tecnológica, com a universalização do acesso aos serviços e informações governamentais, são objeto de estudos e pesquisas. A despeito das desigualdades de renda e de acesso à tecnologia e das dificuldades orçamentárias, o governo pretende ampliar a oferta de informação eserviços públicos pela I nternet.

No caso do Brasil, segundo documento apresentado no seminário U sing knowledge for development: B razil India - China Forum, realizado em Londres, de 19 a 25 demarço de2001, no início de 2001 o governo já contava com 629 serviços e 3.500 tipos de informação com possibilidade de acesso pela Internet. Já o grupo de trabalho N ovas Formas Eletrônicas deI nteração (2000) * registra como diagnóstico inicial a existência de redes isoladas, a falta de padronização nos serviços e a falta de comunicação epermuta de dados entresistemas deórgãos governamentais.

Essas iniciativas demonstram que o governo brasileiro está consciente de sua responsabilidade na atuação para incrementar presença na Internet, em um ambiente seguro, e, ao mesmo tempo, aumentar as oportunidades deacesso às modernas tecnologias. Ainda assim, percebese uma visão fragmentada da ação governamental, em função da ausência ou pouca evidência de atuação baseada em sistemas interorganizacionais.

Aspectos como cooperação, convergência e integração das redes e dos sistemas deinformação do governo devem ser considerados para permitir uma utilização racional dos recursos que a sociedade repassa sob a forma de impostos etaxas. Além disso, éimportante registrar os ganhos potenciais quea integração pode trazer, na forma de economia de escala na aquisição de produtos e serviços de tecnologia.

\footnotetext{
*PROPOSTA de política de governo eletrônico para o Poder Executivo Federal. G rupo de Trabalho: N ovas Formas Eletrônicas de Interação. Brasília, 20 set. 2000 ( N ão publicado).
} 
Sobrea questão orçamentária, a preocupação do governo é medir, física e financeiramente, o esforço dedicado ao incremento do acesso dos cidadãos às informações e serviços públicos. É a tentativa de mensuração do impacto dos custos da sociedade da informação nas contas públicas. Com relação ao assunto, Suaiden (2001) registra a preocupação de que os orçamentos consignados não refletem um crescimento do acesso à informação, embora seja clara a preocupação com questões tecnológicas e de infra-estrutura de comunicação.

Apesar da preocupação registrada com os aspectos externo e econômico da questão, os documentos do Programa Sociedade da I nformação reconhecem que o fundamento da ação governamental deve ser o cidadão brasileiro e seu direito à informação. Segundo Jardim (1999) , o direito à informação situa-se como um direito civil, um direito político e um direito social.

O exercício de tal direito, entretanto, está condicionado às possibilidades de efetivo acesso e à capacidade de entendimento da informação, esão justamente a esses condicionantes queo Programa SociedadedaI nformação pretendeatender. $\hat{E}$, para o Estado brasileiro, o momento de tomar decisões e implementá-las. Propiciar informação e educação a todos os cidadãos não significa, porém, tornar o Estado onipresente e determinador do destino das pessoas. L onge de mediar as relações entre os homens ou de guiá- los, cabe ao Estado prover, ou permitir que outros o façam, o acesso à informação.

M iranda (1977) afirma quea geração de conhecimento acontece através dos canais do sistema educativo, por meio da transmissão da informação. Esses canais poderão ser incrementados a partir da própria infra-estrutura em construção, como pretende o Programa Sociedade da Informação. A educação serátransformada, passando de uma experiência passiva e individual para um novo processo interativo ecoletivo (Sullivan-Trainor, 1995).

A educação, de fato, deve ter como tar efa a capacitação dos homens pela combinação de cidadania com liberdade na criatividadeindividual (Russell, 1979). M iranda (1977) afirma que a capacidade dos cidadãos de julgar e participar do governo também está relacionada à quantidade è qualidade da informação à qual eles têm acesso. M as a informação não precisa apenas ser acessada ou ter sua circulação facilitada, é preciso que ela seja percebida eentendida, e essa capacidade somente pode ser desenvolvida com processos educacionais adequados.

A participação do profissional de ciência da informação no processo de oferta de produtos e serviços mostra-se com crescenteimportância (Rosenfeld \& M orville, 1998).
O s cyberians (termo usado por Rosenfeld e M orville para a nova geração delibrarians) entendem que o novo terreno da I nternetéuma nova fronteira, depois de muito tempo provendo acesso à informação impressa armazenada em bibliotecas tradicionais. Esse conhecimento acumulado, devidamente atualizado em termos tecnológicos, pode ser usado para resolver problemas de informação em inúmeros contextos (Barbosa, 1998).

Em suma, o desafio a ser vencido é manter a simplicidade e, ao mesmo tempo, a funcionalidade, tendo sempreem vista que 0 interesse central é 0 atendimento das necessidades do cidadão, e não a promoção da organização. É contribuir para a ampliação da participação nos processos decisórios do governo, para a compreensão dos processos econômicos, sociais e políticos, assim como seus conflitos inerentes nos níveis nacional einternacional (Somavía, 1980). N esseprocesso deconstrução desistemas de informação, pode-se afirmar que 0 ambiente informacional atual já contempla as expectativas traçadas em 1974 por Servan-Schreiber, quanto ao acesso à informação:

a) qualquer que seja o lugar em que a informação é produzida;

b) no próprio momento em quea informação éproduzida (e, conseqüentemente, influenciando os próprios acontecimentos);

c) onde quer que se encontre o consumidor da informação;

d) qualquer que seja o momento em que se precisar da informação;

e) qualquer queseja o tema desejado.

\section{ESTUDO DE CASO: ORGANIZAÇÕES PÚ BLICAS E SISTEMAS INTERORG AN IZACIONAIS}

\section{M otivações e fatores críticos de sucesso}

A decisão de implementar sistemas interorganizacionais, na esfera pública ou em qualquer tipo de organização, significa uma quebra de paradigma. Partindo do conceito discutido por Kuhn (1982), paradeigma (original grego) é uma forma de entender a realidade, uma forma de pensar um modelo. Assim, afastar-se da forma tradicional de gerenciamento de sistemas e se dispor a entender os benefícios (e riscos) do modelo 


\section{H enrique Flávio R odrigues da Silveira}

colaborativo implica aceitar um novo paradigma, um novo modelo, com outros limites e novas regras.

A resistência a mudanças, ainda segundo K uhn, têm o papel de assegurar que o paradigma anterior não será facilmente abandonado, e a crise decorrente dos conflitos tem por significado exatamente 0 fato de indicar que é chegada a ocasião para renovar profundamente os instrumentos e os conhecimentos existentes (as diferenças entre paradigmas sucessivos são ao mesmo tempo necessárias eirreconciliáveis). Ademais, a transição para um novo paradigma não é uma rearticulação do velho paradigma: é preciso modificar concepções, métodos e objetivos.

A migração para ambientes colaborativos, com a prevalência de sistemas interorganizacionais, é uma mudança de paradigma e, como tal, deve ser encarada pelas organizações: com políticas e estratégias adequadas ao novo ambiente, a partir de motivações claramente identificadas e com respaldo institucional, além de dedicar especial atenção para os fatores críticos de sucesso, que poderão definir os resultados na nova empreitada.

As motivações para o envolvimento de uma organização no planejamento de sistemas interorganizacionais podem ser resumidas em cinco grupos principais:

a) compartilhamento de informações;

b) aumento de produtividade;

c) redução de custos;

d) determinação superior, legal ou normativa;

e) incremento do relacionamento com os clientes/ usuários.

As maiores preocupações das organizações com relação a sistemas interorganizacionais, identificadas como fatores críticos de sucesso, são:

a) custo de desenvolvimento e manutenção;

b) segurança;

c) habilidadee/ ou capacidade de cooperação com outras organizações;

d) efeitos na organização administrativa interna;

e) apoio da alta gerência.

\section{Pressupostos}

Para efeito da pesquisa, admite-se que as motivações e os fatores críticos de sucesso que devem ser considerados no processo de planejamento de sistemas interorganizacionais no setor público para o ambiente Internet correspondem àqueles anteriormente identificados.

Para efeito de levantamento junto às organizações públicas selecionadas, partiu-se do pressuposto geral de que todas as organizações pesquisadas registram participação em sistemas interorganizacionais ou manifestam intenção de participar deles, seja por iniciativa própria ou por determinação superior, legal ou normativa.

Q uanto aos pressupostos específicos, foram levantandos os seguintes:

a) a principal motivação para a participação em sistemas interorganizacionais éo incremento do relacionamento com os clientes/ usuários, levando em conta o novo modelo degestão preconizado pelo governo federal, que coloca o usuário/ cidadão como centro das atenções das organizações públicas;

b) a importância atribuída às motivações "compartilhamento de informações" e "aumento de produtividade" é superior àquela atribuída à motivação "redução de custos", refletindo a consciência das organizações pesquisadas de que não devem se envolver com sistemas interorganizacionais apenas por uma questão de redução de custos, e sim em função da expectativa de melhoria dos processos e dos resultados;

c) o principal fator crítico de sucesso para o planejamento de sistemas interorganizacionais é a segurança, tendo em vista a preocupação que as organizações têm com o sigilo e a preservação dos dados sob suas responsabilidades, inclusive por determinação legal;

d) a importância atribuída aos fatores críticos de sucesso "habilidade e/ ou capacidade de cooperação com outras organizações" e "custo de desenvolvimento e manutenção" é superior àquela atribuída ao fator crítico "efeitos na reorganização administrativa interna", o que indica que a implantação de mudanças administrativas eventualmente necessárias a partir da utilização de 
sistemas interorganizacionais não traz dificuldades maiores que a falta de articulação ou de recursos para o projeto.

\section{M etodologia}

Para coletar os dados e comprovar os pressupostos, optou-sepela aplicação de entrevistas junto a órgãos públicos com área de atuação relacionada com temas de interesse do Banco Central e vinculação hierárquica ao M inistério da Fazenda, em função do relacionamento institucional do autor da pesquisa com o Banco Central do Brasil, vinculado àquela pasta.

Q uatorzeórgãos foram selecionados, dos quais doze ( $86 \%)$ responderam às questões sobre a participação e a percepção das organizações em relação a sistemas interorganizacionais. Cumprindo o que estava disposto quando da coleta dos dados, as respostas foram agrupadas, de forma a tratar os dados sempre em bloco.

A primeira questão dirigida aos órgãos pesquisados indagou sobre 0 envolvimento da organização em relação a sistemas interorganizacionais. Os resultados encontram-se na tabela 1.

Para efeito de verificação junto aos órgãos públicos selecionados e por razões de clareza e concisão, as motivações para o envolvimento de uma organização no planejamento de sistemas interorganizacionais foram sintetizadas em cinco grupos principais. N esse item, os resultados estão apurados na tabela 2.

F oram classificados, também, os fatores críticos de sucesso identificados com maior recorrência de referências na literatura. 0 resultado encontra-se na tabela 3.

Ci. Inf., Brasília, v. 32, n. 2, p. 107-124, maio/ago. 2003

\section{TABELA 2}

\section{TABELA 3}

\section{TABELA 1 \\ E nvolvimento organizacional em sistemas interorganizacionais}

\begin{tabular}{|c|c|}
\hline $\begin{array}{l}\text { Q ual o envolvimento da organização com sistemas } \\
\text { interorganizacionais? } \\
\text { Atenção: as opções (a) e (b) não são excludentes }\end{array}$ & $\begin{array}{l}\text { Quantidade } \\
\text { derespostas }\end{array}$ \\
\hline $\begin{array}{l}\text { a) é patrocinadora de um ou mais sistemas ou projetos de sistemas } \\
\text { (responsável pelo desenvolvimento ou pela iniciativa de } \\
\text { desenvolvimento) }\end{array}$ & $\begin{array}{c}3 \\
(25 \%)\end{array}$ \\
\hline $\begin{array}{l}\text { b) é participante de um ou mais sistemas ou projetos de sistema } \\
\text { (integra o conjunto de organizações participantes de um sistema } \\
\text { desenvolvido ou patrocinado por outra organização) }\end{array}$ & $\begin{array}{c}10 \\
(83 \%)\end{array}$ \\
\hline $\begin{array}{l}\text { c) não é patrocinadora ou participante de sistemas ou projetos de } \\
\text { sistemas, mas há planos de participar de projetos de sistemas } \\
\text { interorganizacionais ou de participar de sistemas já existentes }\end{array}$ & $\begin{array}{c}2 \\
(17 \%)\end{array}$ \\
\hline $\begin{array}{l}\text { d) não é patrocinadora ou participante de sistemas ou projetos de } \\
\text { sistemas, e não há planos de participar de projetos de sistemas } \\
\text { interorganizacionais ou de participar de sistemas já existentes }\end{array}$ & $\begin{array}{c}0 \\
(0 \%)\end{array}$ \\
\hline
\end{tabular}

M otivação para a participação em sistemas interorganizacionais

\begin{tabular}{lc}
\hline $\begin{array}{l}\text { Classifique, da menos importante (1) à mais importante (5), } \\
\text { as motivações para o envolvimento, ou potencial envolvimento, } \\
\text { da sua organização em sistemas interorganizacionais }\end{array}$ & $\begin{array}{c}\text { Somatório das } \\
\text { respostas }\end{array}$ \\
\hline a) compartilhamento de informações & $46(25,6 \%)$ \\
b) aumento de produtividade & $35(19,4 \%)$ \\
c) redução de custos & $24(13,3 \%)$ \\
d) determinação superior, legal ou normativa & $38(21,1 \%)$ \\
e) incremento do relacionamento com os clientes/ usuários & $37(20,6 \%)$ \\
\hline
\end{tabular}

\section{Fatores críticos de sucesso para a participação em sistemas interorganizacionais}

\begin{tabular}{lc}
\hline Classifique, do menos importante (1) ao mais importante (5), & $\begin{array}{c}\text { Somatório } \\
\text { das } \\
\text { os fatores críticos de sucesso para o planejamento desistemas } \\
\text { interorganizacionais, sob o ponto de vista de sua organização }\end{array}$ \\
\hline a) custo de desenvolvimento e manutenção & $39(21,7 \%)$ \\
b) segurança & $52(28,9 \%)$ \\
c) habilidadee/ ou capacidade de cooperação com outras organizações & $41(22,8 \%)$ \\
d) efeitos na organização administrativa interna & $22(12,2 \%)$ \\
e) apoio da alta gerência & $26(14,4 \%)$ \\
\hline
\end{tabular}

\section{Resultados}

As respostas à primeira indagação sobre o envolvimento da organização em relação a sistemas interorganizacionais revelam a percepção, por parte das organizações pesquisadas, da presença desses sistemas em suas atividades. $\mathrm{N}$ enhuma das organizações pesquisadas se declarou, ao mesmo tempo, nãopatrocinadora, não-participante e sem planos de participar de sistemas interorganizacionais. 


\section{H enrique Flávio R odrigues da Silveira}

TABELA 4

\section{Consolidação dos resultados da pesquisa}

\begin{tabular}{|c|c|c|c|c|c|c|c|c|c|c|c|c|c|c|c|c|c|}
\hline motivaḉes & 1 & 2 & 3 & 4 & 5 & 6 & 7 & 8 & 9 & 10 & II & 12 & & & $\begin{array}{l}\text { Desvio } \\
\text { padrăio }\end{array}$ & Moda & $\begin{array}{l}\text { Freqüéncia } \\
\text { da Moda }\end{array}$ \\
\hline compartilhamento & 5 & 1 & 3 & 5 & 4 & 5 & 3 & 4 & 4 & 3 & 4 & 5 & 46 & $25,6 \%$ & 1,19 & 5 & 4 \\
\hline produtividade & 4 & 2 & 1 & 2 & 2 & 2 & 1 & 5 & 3 & 4 & 5 & 4 & 35 & $19,4 \%$ & 1,44 & 2 & 4 \\
\hline reducaio de custos & 3 & 4 & 2 & 1 & 1 & 1 & 4 & 2 & 2 & $i$ & 2 & 1 & 24 & $13,3 \%$ & 1,13 & 1 & 5 \\
\hline ordem superior & 2 & 5 & 4 & 3 & 5 & 3 & 2 & 3 & 1 & 5 & 3 & 2 & 38 & $21,1 \%$ & 1,34 & 3 & 4 \\
\hline relaçào c/cliente & 1 & 3 & 5 & 4 & 3 & 4 & 5 & 1 & 5 & 2 & 1 & 3 & $\begin{array}{r}37 \\
180\end{array}$ & $20,6 \%$ & 1,56 & 1 & 3 \\
\hline $\begin{array}{l}\text { FCS } \\
\text { custo }\end{array}$ & 1 & 5 & 4 & 3 & 2 & 4 & 4 & 4 & 3 & 4 & 2 & 3 & 39 & $21,7 \%$ & 1,14 & 4 & 5 \\
\hline segurança & 5 & 3 & 5 & 4 & 4 & 5 & 3 & 5 & 4 & 5 & 4 & 5 & 52 & $28,9 \%$ & 0,78 & 5 & 6 \\
\hline habilidades & 4 & 2 & 2 & 2 & 3 & 3 & 5 & 3 & 5 & 3 & 5 & 4 & 41 & $22.8 \%$ & 1,16 & 3 & 4 \\
\hline reorganizaçào & 3 & 4 & 1 & 5 & 1 & 1 & 1 & 1 & 1 & 2 & 1 & 1 & 22 & $12,2 \%$ & 1,40 & 1 & 8 \\
\hline apoio superior & 2 & 1 & 3 & 1 & 5 & 2 & 2 & 2 & 2 & 1 & 3 & 2 & $\begin{array}{r}26 \\
180\end{array}$ & $14,4 \%$ & 1,11 & 2 & 6 \\
\hline
\end{tabular}

0 pressuposto geral de que "todas as organizações pesquisadas registram participação em sistemas interorganizacionais ou manifestam intenção de participar deles" foi confirmado. D as organizações consultadas, $100 \%$ declararam participar ou ter intenções de participar de sistemas interorganizacionais.

Dezessete por cento (17\%) das organizações declararam que, apesar denão serem patrocinadoras ou participantes de sistemas ou projeto de sistemas interorganizacionais, têm planos de participar de projetos ou de sistemas já existentes. A decisão de participar de sistemas interorganizacionais pode indicar a consciência da inexorabilidade da presença crescente desses sistemas na rotina das organizações.

O itenta e três por cento (83\%) das organizações se declararam participantes de sistemas interorganizacionais, ainda que não tenham sido por elas patrocinados. Considerando a complexidade e os impactos de sistemas interorganizacionais, pode-se afirmar que as organizações têm consciência da existência desses sistemas. Essa consciência pode significar um espaço para discussão mais elaborada a respeito dos efeitos, benefícios e preocupações relacionados aos sistemas interorganizacionais, a ser desenvolvida em estudos posteriores.

Existe, ainda, a prevalência de sistemas interorganizacionais com gerência centralizada. A penas três, das 12 organizações pesquisadas, declararam-se patrocinadoras desistemas, ao mesmo tempo em que se declararam também participantes. Essa concentração na gestão dos sistemas pode indicar que os sistemas interorganizacionais ora em uso têm um "interessado principal", definido como a organização que seria a maior interessada na execução regular esatisfatória do sistema.

Sobre motivações e fatores críticos de sucesso, a partir dos dados coletados foram calculados - para cada item - o desvio-padrão, como medida de dispersão dos dados em relação à média, a moda, como medida de ocorrência mais freqüente, ea freqüência, como medida de repetição de valores. A tabela 4 foi elaborada com os resultados da pesquisa, para efeito de análise.

A motivação considerada mais importante foi "compartilhamento de informações", com 25,6\% do total de pontos, cinco pontos percentuais acima da segunda motivação mais bem classificada. 0 fato deter apresentado também o segundo menor desvio-padrão indica quehouve certa convergência de opiniões sobre a maior importância do "compartilhamento de informações" como motivação para o envolvimento das organizações com sistemas interorganizacionais. Essa possibilidade foi tratada por D rucker (1971), H ill \& Ferguson (1989), Li \& Williams (1999), M aloff (1996) eZwass (2001).

Ainda que o governo venha desenvolvendo esforços para mudar a cultura da administração pública, deum modelo burocrático (mais voltado para a própria estrutura do estado), para um modelo gerencial (mais voltado para o cliente, para o cidadão), a importância dada ao "compartilhamento de informações", em detrimento da importância atribuída à motivação "incremento do relacionamento com os clientes/ usuários", pode indicar maior atenção aos processos internos. 


\section{M otivações e fatores críticos de sucesso para o planejamento de sistemas interorganizacionais na sociedade da informação}

Com esse resultado, o pressuposto de que a "a principal motivação para a participação em sistemas interorganizacionais éo incremento do relacionamento com os clientes/ usuários, levando em conta o novo modelo de gestão preconizado pelo governo federal, que coloca o usuário/cidadão como centro das atenções das organizações públicas", não foi confirmado.

D e qualquer forma, há percepção, por parte das organizações, da importância dos sistemas interorganizacionais para racionalizar os processos de geração, seleção, representação, armazenamento, recuperação, distribuição e uso da informação, expressa na manifestação do "compartilhamento da informação" como motivação maior para a participação em sistemas interorganizacionais.

A motivação à qual foi atribuída a menor importância foi "redução de custos", com 13,3\% do total, mais de seis pontos percentuais a menos que o quarto lugar. Foi também o item que recebeu o menor desvio-padrão do grupo, o que pode indicar certa concordância entre as organizações. D e fato, a moda para este item foi o valor "1" (menor importância), com a maior freqüência da moda do grupo - cinco em 12 respostas.

Foi confirmado o seguinte pressuposto: a importância atribuída às motivações "compartilhamento de informações" e "aumento de produtividade" é superior àquela atribuída à motivação "redução de custos", refletindo a consciência das organizações pesquisadas de que não devem se envolver com sistemas interorganizacionais apenas por uma questão de redução de custos, e sim em função da expectativa de melhoria dos processos e dos resultados.

M enor atribuição de importância aos custos, em termos relativos a outras opções, pode indicar que, mesmo em época de restrições orçamentárias, as organizações entendem que o envolvimento com sistemas interorganizacionais não deve ser induzido por questões de custos, e sim devido às possibilidades que o novo ambiente colaborativo pode representar. Sobre custos, diversos autores registraram considerações, entre eles Bowonder (apud M oresi, 2000), Bryonjolfsson (2001), Claver, Garcia \& Gonzaléz (2001) eD avenport (1994).

O segundo lugar para a motivação "determinação legal, superior ou normativa", com $21,1 \%$ do total, pode denotar a influência da questão burocrática e formal que delimita os espaços de atuação das organizações públicas. É necessário registrar, porém, que as organizações pesquisadas atuam na área do M inistério da F azenda, queé fortemente regulamentada, sendo que alguns sistemas têm amparo legal para sua execução. A motivação com base em determinação externa foi especialmentetratada em C rook \& K umar (1998).

A motivação "incremento do relacionamento com os clientes/ usuários" ficou em terceiro lugar, com $20,6 \%$ do total, a apenas $0,5 \%$ do segundo colocado. A importância atribuída a essa motivação pode ser considerada como uma tomada de consciência, por parte das organizações, da necessidade de atender melhor ao cliente/ usuário/ cidadão.

Foi o item, entretanto, que apresentou mais diversidade de opiniões, representado pelo maior desvio-padrão do grupo e pela menor freqüência da moda, o que pode significar queas organizações têm diferentes percepções da importância do relacionamento com os clientes, de acordo com suas funções e estruturas. Também nesse ponto poderiam ser desenvolvidos estudos posteriores, talvez agrupando as organizações por perfil de relacionamento com o público. A motivação com base no cliente foi enfatizada em especial por Fleming (1998), Guimarães (2001) eJ ardim (1999).

O "aumento de produtividade" esperado com o envolvimento da organização em sistemas interorganizacionais ficou em quarto lugar, com 19,4\%. 0 fato deo "aumento de produtividade" apresentar mais de seis pontos percentuais à frente da motivação "redução de custos" pode significar que as organizações entendem que 0 aumento de produtividade contribui mais para 0 sucesso da organização quea busca da redução de custos.

Sobre isso pode-se questionar também o grau de consciência das organizações quanto ao fato de que sistemas interorganizacionais podem não gerar redução de custos, podendo mesmo implicar maiores gastos em decorrência dos recursos e tecnologias a serem empregados, e, dessa forma, a opção pelo ambiente colaborativo somente se justifica como um apelo à melhor performance das organizações. A busca de aumento de produtividade como motivação para a cooperação foi tratada em especial por Andrews (1983), Cunha (1999), D rucker (1963), Tarapanoff \& Álvares (1994) eVancil (1983).

Q uanto aos fatores críticos de sucesso, a primeira colocação em importância ficou com o fator "segurança", com $28,9 \%$ do total, mais de sete pontos percentuais acima do segundo colocado. 0 fato de também ter 


\section{H enrique Flávio R odrigues da Silveira}

apresentado o menor desvio-padrão do grupo e o único abaixo de um $(0,78)$, uma moda de valor " 5 ", apoiada em uma freqüência alta ( $50 \%$ do total de respostas), indica clara compreensão, por parte das organizações, da importância que deve ser atribuída ao aspecto "segurança" em um sistema interorganizacional.

Com os resultados, confirmou-se o pressuposto: "o principal fator crítico desucesso para o planejamento de sistemas interorganizacionais é a segurança, tendo em vista a preocupação que as organizações têm com o sigilo ea preservação dos dados sob sua responsabilidade, inclusive por determinação legal". Destaque-se, especificamente no caso das organizações pesquisadas, que há exigências legais e regulamentares sobre o sigilo das informações que comprometem os gestores em relação à guarda e proteção dos dados. Sobre segurança, diversos autores destacaram sua importância, entre eles Clarke (1992), D 'Andréa (2001), D avis \& D avidson (1993) eM artini (2000).

0 reconhecimento de que a participação em sistemas interorganizacionais requer "habilidade e/ ou capacidade de cooperação com outras organizações" fez com que esse fator ficasseem segundo lugar, com 22,8\% do total. D efato, o processo de construção e de gestão de sistemas que envolvem várias organizações requer uma disposição para o aprendizado contínuo, para a negociação e para o trabalho em grupo. Reconhecendo a importância desse fator, as organizações dão importantepasso para o sucesso de sua participação em sistemas interorganizacionais, como destacado por Ciborra (1993), D rucker (1971, 1996), G ates (1999), Pouloudi \& Reed (1998) ePreiss, Goldman \& Nagel (1998).

Se a questão de "redução de custos" ficou em último lugar como motivação para o envolvimento das organizações em sistemas interorganizacionais, o fator crítico de sucesso "custo de desenvolvimento e manutenção" representa uma preocupação maior para as organizações pesquisadas, ficando em terceiro lugar, com $21,7 \%$ do total, apenas $1,2 \%$ abaixo do segundo colocado. As restrições à gestão orçamentária e financeira das organizações públicas, que exigem procedimentos específicos para aquisição de produtos e serviços, bem como para assinatura de contratos, podem levar as organizações a refletir, com bastante propriedade, sobre os encargos decorrentes da participação em sistemas interorganizacionais.
Os resultados confirmaram o pressuposto de que "a importância atribuída aos fatores críticos de sucesso "habilidade e/ ou capacidade de cooperação com outras organizações" e "custo de desenvolvimento emanutenção" é superior àquela atribuída ao fator crítico "efeitos na reorganização administrativa interna", o que indica quea implantação de mudanças administrativas eventualmente necessárias a partir da utilização de sistemas interorganizacionais não traz dificuldades maiores quea falta de articulação ou de recursos para o projeto.

O "apoio da alta gerência" foi considerado pelas organizações pesquisadas o quarto fator em ordem de importância, com 14,4\% do total, mais de sete pontos percentuais abaixo do terceiro lugar. Por envolver interesses e recursos de diferentes organizações etambém pelos impactos nos processos internos que um sistema interorganizacional pode acarretar, o apoio da alta gerência é fundamental. A posição relativa desse fator na pesquisa ora sob análise poderia ser explicada pelo fato deque os comandos das organizações já se envolvem necessária e decisivamente no projeto de sistemas interorganizacionais, tendo sido atribuída importância relativa maior aos outros fatores (segurança, habilidades e custo). Entre outros autores, Rosenfeld \& M orville (1998) destacaram a relevância desse fator no desenvolvimento desistemas interorganizacionais.

Finalmente, os "efeitos na organização administrativa interna" decorrentes da participação em sistemas interorganizacionais ficaram com aúltima posição: 12,2\% do total. Porém, o fato de ter registrado o maior desviopadrão do grupo, bastante superior aos demais, euma moda de valor "1", com a maior freqüência de moda do grupo (oito ocorrências em 12 resultados), indica queesses efeitos são avaliados por algumas organizações de forma bastante diferente de outras, talvez em função de suas características e cultura organizacional, certamente por conta dos impactos dereestruturação causados pela opção por sistemas interorganizacionais. A necessidade de implementar mudanças na organização em decorrência da adoção de um ambiente informacional colaborativo foi objeto de estudo por parte de Adernes (2001), Chavetando (1998), Cruz (1998) eG rabowski \& Roberts (2001), entreoutros.

0 único pressuposto não confirmado diz respeito ao incremento do relacionamento com o cliente/ usuário como motivação preponderantena decisão de participar de sistemas interorganizacionais. A não-confirmação desse pressuposto poderia indicar que as organizações 


\section{M otivações e fatores críticos de sucesso para o planejamento de sistemas interorganizacionais na sociedade da informação}

pesquisadas desenvolvem a maior partede seus processos internamente, sendo seus resultados aferidos não a partir de avaliação externa, mas do cumprimento regular de suas obrigações.

Entretanto, o desvio-padrão para a motivação em pauta foi o mais alto do grupo, e, apesar de a moda ter sido o valor "1" (menor importância), sua freqüência foi a mais baixa do grupo. Estes indicadores permitem afirmar que não houve convergência de opiniões sobre a questão, 0 que denota o fato de que as organizações percebem a importância do incremento do relacionamento com os clientes/usuários de forma diversificada.

Em resumo, a análise dos dados indica que as organizações têm a percepção da presença e da importância dos sistemas interorganizacionais em suas atividades. As motivações parecem estar mais ligadas a aspectos de melhoria da performance organizacional (compartilhamento e relação com o cliente), que a aspectos meramente relacionados à redução de custos. $\mathrm{H}$ á clara percepção da necessidade de implementar requisitos de segurança como fator crítico de sucesso para o desenvolvimento e gerência de sistemas interorganizacionais, sendo menos relevantes os aspectos ligados à estrutura interna das organizações.

0 fato de as freqüências de moda dos fatores críticos de sucesso terem sido, no conjunto, mais altas que as freqüências de moda das motivações, de forma inversa aos desvios-padrões, podeindicar quehá menor influência das características de cada organização na forma de avaliar os fatores críticos de sucesso, sendo a percepção da importância das motivações mais influenciada pelas características próprias de cada organização.

\section{CONCLUSÕES}

"A maior descontinuidade que nos cerca éa mudança ocorrida na posição e no poder do conhecimento"

(D rucker, 1969)

0 estudo de sistemas interorganizacionais demonstra que se trata de um fenômeno que deve ser seriamente considerado pelas organizações, em suas múltiplas facetas. O s executivos precisam estar preparados para lidar com as conseqüências organizacionais de sua implementação, para além das preocupações estritamentetécnicas.

Assim, quando o tema "sistemas interorganizacionais" assume importância para todas as organizações, pelas responsabilidades e possibilidades queencerra, a ciência da informação tem papel fundamental no estudo das necessidades informacionais, do comportamento na busca da informação, no estudo do uso da informação e dos processos de interação e planejamento dos sistemas deinformação.

A mudança tecnológica de caráter revolucionário exerce coerção sobre as organizações - ela exigeinovação. N ão se trata de apenas aperfeiçoar os processos, mas de repensar a própria organização. D rucker (1971) alerta que nada poderia ser menos produtivo do que tornar mais eficiente aquilo que não deveria estar sendo feito. As perguntas devem ser sobre quais as tarefas que devem ser executadas e por que devem sê-lo.

Para sobreviver e prosperar em um ambiente turbulento, torna-se crítico gerenciar o conhecimento de fora para dentro, e não apenas de dentro para fora. É preciso ampliar a colaboração, o compartilhamento, 0 aprendizado, para além das fronteiras da organização. A value chain (cadeia de valor) em que a organização está inserida está mudando muito rapidamente, euma das mais poderosas forças que movem (e provocam) as mudanças éa tecnologia da informação e suas aplicações.

U ma nova organização deve ser fundada em um ambiente onde as organizações e as pessoas estão conectadas em rede (internetworking), onde a colaboração deverá suplantar a rivalidade e a competição predatória, como chave para um desenvolvimento sustentável dos negócios e da sociedade. Convênios para ações cooperativas, descritas por Cunha (1999) para bibliotecas, poderão se tornar usuais entre organizações públicas.

Afinal, se ignorarmos as fronteiras das organizações e repensarmos o trabalho retroativamente a partir do seu resultado final, ou seja, começando com a satisfação do cliente, freqüentemente veremos que o processo que produz aquele resultado envolvevárias organizações, cada uma executando uma parte do processo.

H ammer (1997) afirma quea essência do gerenciamento de um negócio é o gerenciamento de seus processos. Seria factível admitir, então, que não caberá mais às organizações gerenciar apenas seus próprios processos, mas participar ativamente da gestão dos processos da cadeia produtiva em que estão envolvidas, o que estaria de acordo com Chiavenato (1998), que destaca a crescente importância da administração sem fronteiras.

O s resultados dos sistemas interorganizacionais podem ser medidos pelo incremento dos resultados financeiros, decorrentes da redução de custos e aumento de produtividade, e pelo aumento na utilidade social das 


\section{H enrique Flávio R odrigues da Silveira}

informações. Entendem-se por aumento da utilidade social os benefícios para a sociedade, advindos da racionalização na utilização dos recursos e da oferta de informações e serviços de forma integrada.

Para Servan-Schreiber (1974), a informação compartilhada encontra-se na base de toda a "boa inteligência", e, assim, se um povo está suficientemente informado, delibera. M esmo a ocorrência de pequenas diferenças não tira do resultado o caráter de "vontade geral" e, por conseguinte, benéfico para o conjunto da sociedade. Thomas efferson (apud Dizard, 1982) também afirmava que a liberdade de expressão e de opinião éa base de todo governo e também que a melhor maneira de aperfeiçoar as imperfeições da democracia é aumentando a quantidadee a qualidade das informações à disposição do povo.

O s estudos aqui apresentados revelam que sistemas interorganizacionais serão decisivos para ampliar a presença do Estado na Internet, facilitando o exercício da cidadania, etambém para aumentar a competitividade das empresas. D eve haver a percepção de que, no setor privado, se uma organização explora o monopólio em um dado segmento, este segmento perderá competitividade e sofrerá abalos na confiança dos consumidores, e, no setor público, a sociedade não aceitará a falta de integração que aumenta o consumo de recursos públicos e reduz a efetividade da ação do Estado. Pode-se afirmar que restarão dois tipos de organização: as colaborativas e aquelas em extinção.

As motivações identificadas na revisão de literatura (compartilhamento de informações; aumento de produtividade; redução decustos; determinação superior, legal ou normativa eincremento do relacionamento com os clientes/usuários) atendem às inquietações dos gestores quanto à racionalização do uso de recursos eao aumento de produtividade, assim como aos anseios da sociedade pelo acesso mais fácil à informação pública integrada.

Q uanto aos fatores críticos de sucesso identificados na revisão de literatura (custo, segurança, habilidade de cooperação, efeitos internos, apoio superior), pode-se afirmar que há suficiente desenvolvimento tecnológico para minimizar as questões relativas à segurança, confidencialidadee integridade das informações, a custos compatíveis com os benefícios esperados. 0 necessário apoio da alta gerência existee pode ser comprovado pelas inúmeras iniciativas governamentais de estímulo à adoção de melhores práticas para o setor público.

Já os fatores relacionados aos efeitos na reorganização administrativa interna, por conta da implementação de sistemas interorganizacionais, devem ser tratados em um contexto de reforma do setor público, no qual há a preocupação com a busca de resultados da ação governamental, com as estruturas internas das organizações públicas em constante aperfeiçoamento, valorizando as competências individuais e o trabalho em equipe, mas sempre com o foco no cliente do governo: a sociedade.

O fator crítico "habilidade e/ ou capacidade de cooperação com outras organizações" parece ser o que vai requerer atenção especial por parte do governo. A persistência de ilhas na administração pública éuma realidade que não pode ser desconsiderada, apesar das iniciativas oficiais conclamando à integração. A mudança cultural dos processos de gestão, a fim de incorporar práticas colaborativas, deve ser estimulada e talvez até ser objeto de mensuração do desempenho dos gestores públicos.

A pesquisa realizada confirma a necessidade crescente de disseminação cooperativa da informação. Já na década de 1970, M iranda (1977) afirmava que os países em desenvolvimento precisavam acelerar a disseminação da informação em todos os níveis de sua estrutura social. Essa éa questão central: assegurar o acesso à informação, para a construção de uma cidadania plena, a activae civitatis (cidadania ativa), de que nos fala Bobbio (1986), pois informação éenergia para mente (D rucker, 1971).

"Ser informado éser livre" ( $N$ orbert Wiener)

A rtigo recebido em 13-02-2003 eaceito para publicação em 07-05-2003 


\section{M otivações e fatores críticos de sucesso para o planejamento de sistemas interorganizacionais na sociedade da informação}

\section{REFERÊ N C IAS}

AGÊNCIANACIONAL DE TELECOMUNICAÇÕES (Brasília, DF) Brasil: uma proposta para o desenvolvimento da cidadania. Brasília, 1999.

ALBERTIN, Alberto Luiz. Administração de informática: funções e fatores críticos de sucesso. São Paulo : Atlas, 1999.

ANDREWS, Kenneth R. Directors' responsability for corporate strategy. In: H AM ERM ESH , Richard. (O rg.). H arvard business review: strategic management. N ew York : John Wiley, 1983. p. 512-521.

BARBOSA, Ricardo R. M onitoração ambiental: uma visão interdisciplinar. Revista de Administração, v. 32, n. 4, p. 42-53, out./ dez. 1997.

Perspectivas profissionais e educacionais em biblioteconomia e ciência da informação. Ciência da Informação. v. 27, n. 1., p. 5360. jan./abr. 1998.

BENIGER, James R. The control revolution. Cambridge : H arvard University, 1986.

BERT AN LAN FFY, L udwig Von. Teoria G eral dos sistemas. Tradução de Francisco M. Guimarães. Petrópolis : Vozes, 1977.

BIO, Sérgio Rodrigues. Sistemas de informação: uma visão gerencial. São Paulo : Atlas, 1996.

BOBBIO, N orberto. A teoria das formas de governo. Tradução de Sérgio Bath. Brasília : U niversitária de Brasília, 2000.

0 futuro da democracia: uma defesa das regras do jogo. Tradução de M arco Aurélio N ogueira. Rio de Janeiro : Paz e Terra, 1986. 171p.

BRASIL. M inistério do Planejamento, O rçamento e G estão. Secretaria de Logística e Tecnologia da Informação. A política de governo eletrônico no Brasil. In: USING KNOWLEDGE FOR DEVELOPMENT: BRAZIL, INDIA, CHINA FORUM, 2001, Wilton Park, UK. Proceedings... Wilton Park: World Bank Institute, 2001.

BRYONJO LFSSON, Erik et al. An empirical analysis of the relationship between information technology and firm size. D isponível em: < http:/ / ccs. mit.edu/papers/ccswp123/ccswp123.html> . Acesso em: 15 mar. 2001.

CH IAVEN ATO, I dalberto. O s novos paradigmas: como as mudanças estão mexendo com as empresas. São Paulo : Atlas, 1998.

$\mathrm{CHOO}, \mathrm{C}$. W. Information management for the intelligent organization. M edford, NJ : Information Today, 1995.

The knowing organization: how organizations use information to construct meaning, create knowledge and make decisions. International Journal of Information M anagement, v. 16, n. 5 , p. 239-340, 1996.

CIBORRA, Claudio. Teams, market and systems: business innovation and information technology. Cambridge : Cambridge University, 1993.

CLARKE, Roger. Extra-organizational systems: a challenge to the software engineering paradigm. In: IFIP W ORLD CONGRESS, 1992, M adrid. Proceedings... M adrid : [s. n.], 1992.

CLAVER, Enrique; GARCIA, D aniel; GONZALÉZ, M. de los Reyes. Interorganizational relationships in the Information era. Disponível em: < http:// inforge.unil.ch/ cimre96/ papers/17/ paper.htm>. Acesso em: 16 mar. 2001.

CONSELH O NACIONAL DE CIÊNCIA E TECN OLOGIA (Brasília, DF). Sociedade da informação. Brasília, 1999.
CORNACH IONE JÚ N IOR, Edgard. Informática aplicada às áreas de contabilidade, administração e economia. São Paulo : Atlas, 1998.

CRO O K, Connie W.; KU M AR, Ram L. Eletronic data interchange: a multi-industry investigation using grounded theory. Information \& M anagement, n. 34, p. 75-89, 1998.

CRUZ, Tadeu. Sistemas de informações gerenciais: tecnologia da informação e a empresa do século XXI. São Paulo : Atlas, 1998.

CU N H A, M urilo B. Desafios na construção de uma biblioteca digital. Ciência da Informação, Brasília, v. 28, n. 3, p. 257-268. set./dez. 1999.

D 'AN D RÉA, Edgar. Security watch. Information Week, v. 3, n. 4, 17 mar. 2001

DAVIS, Stan; DAVIDSO N, Bill. Visão 2020: administrando a sua empresa hoje para vencer amanhã. Tradução de Waltensir Dutra. Rio de Janeiro : Campus, 1993.

DIZARD, Wilson P. The coming information age. N ew York : Longman, 1982.

DRUCKER, Peter $F$. M anaging for business effectiveness. In: H AM ERM ESH, Richard. (O rg.). H arvard business review: strategic management. $\mathrm{N}$ ew York : John Wiley, 1983. p. 65-76.

Administrando para o futuro: os anos 90 e a virada do século. Tradução de N ivaldo M ontigelli Júnior. São Paulo : Pioneira, 1996.

Tecnologia, gerência e sociedade. Tradução de Luiz Carlos Lucchetti Gondim. Petrópolis, RJ : Vozes, 1971.

U ma era de descontinuidade. Tradução de J. R. Brandão Āzevedo. São Paulo : Círculo do Livro, 1969.

Além da revolução da informação. HSM M anagement, v. 18, p. 48-55, jan./fev. 2000.

\section{0 .}

O futuro já chegou. Exame Digital, v. 34, n. 6, 22 mar.

EIN-DOR, Philip; SEGEV, Eli. Administração de sistemas de informação. Tradução de M arina Cunha Brenner. Rio de Janeiro : Campus, 1986.

FLEM IN G, Jennifer. Web navigation: designing the user experience. Washington, DC : O'reilly, 1998.

GATES, Bill. A empresa na velocidade do pensamento: com um sistema nervoso digital. Tradução de Pedro M aia Soares e Gabriel Tranjan N eto. São Paulo : Companhia das Letras, 1999.

GRABO WSKI, M artha; ROBERTS, Karlene. Risk mitigation in virtual organizations. Journal of Computer-M ediated Communication, v. 3, n. 4, June 1998. Disponível em: < http:/ / www. ascusc.org/jcmc/ vol3/ issue4/grabowski.html> . Acesso em: 16 mar. 2001.

GUIM ARÃES, Camila. Todos juntos: a empresa sem porteiras. Exame Negócios, v. 2, n. 6, jun. 2001.

H AM M ER, M ichael. Além da reengenharia. Tradução de Ana Beatriz Rodrigues e Priscilla M artinds Celeste. Rio de Janeiro : Campus, 1997.

HILL, N. C.; FERGUSON, D. M. Electronic Data Interchange: a definition and perspective. EDI Forum: The Journal of Electronic D ata Interchange, v. 1, 1989.

KUH N, Thomas S. A estrutura das revoluções científicas. Tradução de Beatriz Vianna Boeira e N élson Boeira. São Paulo : Perspectiva, 1982. 


\section{H enrique Flávio R odrigues da Silveira}

LI, Feng; WILLIAM S, H oward. New collaboration between firms: the role of interorganizational systems. In: HAWAII INTERNATIONAL CONFEREN CE ON SYSTEM SCIENCES, 32., Big Island, H awaii, 1999. Proceedings... H awaii : 1999.

M ALO FF, Joel. A Internet e valor da internetização. Tradução de Rose N ânie H eringer da Silva. Internet World M agazine, jul./ago. 1996.

MARTIN I, Renato. Echelon: alguém te escuta. Revista do Linux, dez. 2000.

MIRANDA, Antônio. Informação para o desenvolvimento. Rio de Janeiro : Livros Técnicos e Científicos, 1977. 135 p.

PEREIRA, Luiz C. B.. A reforma do Estado dos anos 90: lógica e mecanismos de controle. Brasília : M inistério da Administração Federal e Reforma do Estado, 1997.

PONJUÁN DANTE, Glória. Gestion de informacion en las organizaciones: principios, conceptos y aplicaciones. Santiago, Chile : Centro de Capacitación en Información, Universidad de Chile, 1998.

POULOUDI, Athanasia ; REED, Chris. Towards a multi-agent representation of stakeholder interests. In: INTERNATIONAL CONFERENCE ON THE PRACTICAL APPLICATIONS OF AGENTS AN D M ULTI-AGENT SYSTEM S: PAAM , 3., 1998, London. Proceedings... London : [s. n.], 1998.

PREISS, Kenneth; GOLDM AN, Steven; NAGEL, Roger. Cooperar para competir: construindo parceiras eficazes. Tradução de $M$ aria Imilda da Costa e Silva. São Paulo : Futura, 1998.

ROCKART, J. F. Chief executives define their own data needs. H arvard Business Review, v. 57, n. 2, p. 81-93, mar./abr. 1979.

ROSENFELD, Louis; M ORVILLE, Peter. Information architecture for the World Wide Web. Washington, DC : O'reilly, 1998.

RUSSELL, Bertrand. 0 poder: uma nova análise social. Tradução de $\mathrm{N}$ athanael C. Caixeiro. Rio de Janeiro : Zahar, 1979.

SERVAN-SCHREIBER, Jean-Louis. O poder da informação. [S. I.] : Publicações Europa-América, 1974.
SH IM IZU, Tamio. Processamento de dados: conceitos básicos. São Paulo : Atlas, 1994.

SILVEIRA, H enrique. D esenvolvimento regional no limiar do século XXI. Fortaleza : Banco do Nordeste, 1996.

Sistemas de informação: uma visão crítica. In: EN CONTRO DE INFORMÁTICA E GESTÃO PÚBLICA, 2., 1993, Salvador. Anais... Salvador : SUCESU, 1993.

Análise SWOT. In: ENCONTRO DA ASSOCIAÇÃO NACIOONAL DE PESQUISA E PÓS-GRADUAÇÃO EM CIÊNCIA DA IN FO RM AÇÃO , 4., 2000, Brasília. Anais... Brasília : EN AN CIB, 2000.

SUAIDEN, Emir J. A biblioteca pública no contexto da sociedade da informação. Ciência da Informação, Brasília, v. 29, n. 2, p. 52-60, maio/ago. 2000.

SU LLIVAN -TRAIN O R, M ichael. Information superhighway. Tradução de L enke Peres Alves de Araújo. São Paulo : M akron Books do Brasil, 1995.

TARAPAN OFF, Kira. Inteligência organizacional e competitiva. Brasília: Universidade de Brasília, 2001.

Técnicas para tomada de decisão nos sistemas de informação. Brasília : Thesaurus, 1995.

; ÁLVARES, Lilian. Cenários para serviços informacionais. Brasília : Thesaurus, 1994.

VICKERY, Brian; VICKERY, Alina. Information science in theory and practice. London : Bowker-Saur, 1989.

WILSON, Tom D. Information management. In: INTERN AT IO N AL ENCYCLOPEDIA OF INFORM ATION AND LIBRARY SCIENCE. London : Routledge, 1997. p. 187-196.

YOURDON, Edward. Análise estruturada moderna. Tradução de Dalton Conde de Alencar. Rio de Janeiro : Campus, 1990.

ZWASS, Vladimir. Structure and macro-level impacts of electronic commerce: from technological infra-structure to electronic marketplaces. Disponível em: < http:// commerce.concordia.ca/ gkersten/ebusiness/ ecom_zwass.html>. Acesso em: 15 mar. 2001. 